\title{
The role of cytokines in the pathogenesis of bronchial asthma and the possibilities of anti- cytokine therapy
}

Keywords: cytokine, sensitization, allergy, mild bronchial asthma, moderate bronchial asthma, moderate bronchial asthma in combination with chronic obstructive pulmonary disease, chronic obstructive pulmonary disease, community-acquired pneumonia, immunoglobulin, allergen

Abbreviations: CK, cytokine; MBA, mild bronchial asthma; MoBA, moderate bronchial asthma; MoBA $+\mathrm{COPD}$, moderate bronchial asthma in combination with chronic obstructive pulmonary disease; CAP, community-acquired pneumonia; HT, hypertension; IHD, ischaemic heart disease; IL, interleukin; IFN $\gamma$, interferon gamma; TNF $\alpha$, tumour necrosis factor alpha; INFP, infectious potential; ATP, atopic potential

\section{Summary}

The study involved 210 people, of whom 32 had MBA, 39 had MoBA, 30 had MoBA+COPD, 38 had COPD, 17 had CAP, 25 had HT and IHD (comparison group) and 20 were healthy subjects. All patients signed informed consent before the study. The Protocol of the study was passed review and was approved by the local ethics Committee of our University. The presence of IgE was determined to tick and house dust allergens, as well as the combined allergens of grass pollen, trees, weeds and flowers, S. pneumon, H. influenzae, and $N$. rerflava. The following cytokines were investigated: IL-4, IL-6, IL-10, IL-17, IFN $\gamma$, TNF $\alpha$. All patients were examined during disease exacerbation. The INFP and ATP were determined for each subject. The results of the study of CK levels and CK combinations, the socalled cytokine profile, indicate that they cannot be used for clinical diagnosis, including nosological diagnosis, the assessment of disease severity, and for selecting individual therapy, including anti-cytokine medications.

The incredible heterogeneity of bronchial asthma (BA) is becoming more and more clear. More than 30 years ago, in 1977, we pointed out the existence of seven clinical and pathogenetic types of BA in patients, subsequently called the following phenotypes: infection-dependent, atopic, hormonal, neuropsychological, autoimmune, significant adrenergic imbalance, and primary bronchial hyperreactivity. ${ }^{1}$ Individual methods of diagnosis and treatment were developed for each of these options, and the results of their testing have been repeatedly published. Subsequently, the phrase "primary bronchial hyperreactivity" was replaced with the idea of exercise-induced BA, peri-menstrual and aspirin-related clinical and pathogenetic types (phenotypes) of BA. The methods of individual diagnosis and treatment continued to improve and their efficacy continued to increase.

Inhalation corticosteroids (ICS) are considered to be the most effective in the treatment of BA. However, in $5-10 \%$ of patients with BA, comprehensive treatment is ineffective even with the inclusion of ICS. ${ }^{2}$ Although the percentage of patients resistant to ICS, is quite low, they make up $50 \%$ of the overall cost of treatment for

\author{
Volume 4 Issue 3 - 2017
}

\section{Fedoseev GB, Trofimov VI, Negrutsa KV, Timchik VG, Golubeva VI, Aleksandrin VA, Razumovskaya TS, Kryakunov KN \\ MV Chernorutsky Department of Hospital Therapy, Pavlov First State Medical University of St Petersburg, Russia}

Correspondence: Fedoseev GB, MV Chernorutsky Department of Hospital Therapy, Pavlov First State Medical University of St Petersburg, Russia, Email fedoseevsp@mail.ru

Received: November 27, 2016 | Published:July 27, 2017

patients with BA. ${ }^{3}$ The insufficient treatment efficacy of existing methods for a group of patients with BA is a reason for examining the BA phenotypes and the endotypes that form them. Knowledge of the mechanisms (endotypes) that form the phenotypes creates the prospects for developing new treatment methods, which take into account the individual characteristics of BA in a particular patient. A cluster analysis of two large European cohorts identified two phenotypes. The first refers to patients with early onset allergic BA, while the second comprises mainly women with late onset disease, without atopy, and with a high body mass index. ${ }^{4}$

Seven parameters were selected to classify the endotypes in patients with BA after the cluster analysis: clinical characteristics, biomarkers, lung physiology, genetics, histopathology, epidemiology, and response to treatment. The following BA endotypes were proposed based on these parameters: 1. Aspirin-sensitive asthma, 2. Allergic asthma, 3. Asthma in preschool children with wheezing, 4. Severe late-onset hypereosinophilic asthma, 5. Asthma in crosscountry skiers. ${ }^{5}$ Another recent study of the type II immune response in patients with BA revealed three phenotypes: 1. Eosinophilic inflammation 2. Allergic sensitisation to antigen-specific $\operatorname{IgE}$ and hyperreactivity, and 3 . Bronchial remodeling. ${ }^{6}$

It is obvious that the above and other similar phenotypes and endotypes do not create the conditions for developing new, personalized treatments. Markers, characterizing the pathogenesis mechanisms, and endotypes that form the different phenotypes of BA, are required, treatment of which would have a therapeutic effect. The cytological characteristics of induced sputum were used as a marker determining the nature of inflammation in patients with BA. The respiratory inflammatory process in patients with $\mathrm{BA}$ is heterogeneous, and the diagnosis of various BA inflammatory phenotypes provides an opportunity to determine the pathogenetic type of BA in a particular patient, thus opening the way for individualized therapy. JL Simpson et al. ${ }^{7}$ have developed four BA inflammatory phenotypes: neutrophilic, eosinophilic, granulocytic and pauci-granulocytic, with 
phenotype combinations also possible. We believe that epithelial and macrophagic phenotypes should also be added to these cellular phenotypes. ${ }^{8}$ At the present time, 161 biomarkers have been described, indicating the presence, severity and nature of airway inflammation in allergic diseases. ${ }^{9}$ Cytokines have been recently used as these kinds of markers, which participate in immunological and other reactions, and are among the mechanisms that generate endotypes of different BA phenotypes.

\section{Main points about cytokines}

Cytokines are small proteins that perform signaling between cells and are a group of humoral factors in both innate and adaptive immunity. The communication that cytokines perform between cells is necessary for various functions (growth, chemo attraction, cell proliferation and differentiation, etc.). The combination and excessive activity of cytokines and pleiotropy make it difficult to assess their role in the pathogenesis of various pathological conditions. Like other inflammatory mediators, cytokines have a high affinity for cell membrane receptors. ${ }^{10}$

\section{Cytokines can be divided into three main groups according to their mechanism of action}

a. Pro-inflammatory, participating in the inflammatory response (interleukins 2, 6, 8, TNF $\alpha$, interferon gamma, etc.),

b. Anti-inflammatory, reducing the development of inflammation (interleukins 4,10 , TGF- $\beta$, etc.),

c. Cellular and humoral immunity regulators (natural or specific) with specific functions (antiviral, cytotoxic, etc.). ${ }^{11}$

\section{Cytokines are divided into three groups according to their functional direction}

1. Cytokines involved in cytotoxic (antiviral and anticancer) humoral and cellular reactions (Th1 and Th17)

2. Cytokines involved in allergic reactions (Th2),

3. Cytokines participating in immunosuppressive and regulatory reactions (Treg). ${ }^{12}$

Due to the significant pleiotropy, the functional direction and mechanisms of action of cytokines can manifest differently under different operating conditions. In experimental models of asthma and according to the results of patient studies, it was found that Th2 cells induce BA through an array of cytokines (IL-4, -5, -9, -10, -13, -25), which activate inflammation of the respiratory tract, either directly or indirectly. ${ }^{13}$ The sum total of the published data on the role of cytokines allows us to identify the main positions that characterize the involvement of cytokines in immune responses: promoting the recognition of antigens, assisting in the expression of adhesion molecules on immune cells, affecting the migration of immune cells, activating monocytes and macrophages, and being antigen co-factors in the activation and proliferation of lymphocytes.

\section{Known substances that have an inhibitory effect on cytokines}

1. cytokine synthesis inhibitors - glucocorticosteroids, cyclosporine A, tacrolimus, and mycophenolate; 2 . humanized antibodies that inhibit cytokines and their receptors; 3. soluble cytokine receptor inhibitors; 4. antagonists and medications that block cytokine transduction. ${ }^{14}$ Cytokines are produced by different immune and nonimmune cells (lymphocytes, epithelial and endothelial cells). ${ }^{11}$

\section{The interaction between cytokines and eosinophils}

The maturation, mobilization and survival of eosinophils in the airways is determined by IL-3 and GM-CSF, but especially by IL-5, which plays a key role in mediating between eosinophils and other cells in the airways, and the formation of eosinophilic inflammation in patients with BA. ${ }^{15} \mathrm{IL}-5$, IL-3 and GM-CSF promote the formation of eosinophils from CD34 hematopoietic progenitor cells, although only IL-5 has a specific effect on eosinophils during development and differentiation. ${ }^{6}$

\section{Eosinophils participate in the generation of cytokines}

1. Eosinophils activate Th2 cells, which secrete cytokines, 2. Eosinophils induce the secretion of cytokines by Th2 cells..$^{13}$ There is growing evidence that eosinophilic inflammation is a sign of BA, and its formation is associated with elevated levels of IL-5 in the bronchi of these patients. ${ }^{16}$ The mechanisms of the eosinophilic type of BA include stimulation of Th2 by allergens, which is accompanied by the release of cytokines (IL-4, -5, -9, -13). Biopsy samples of bronchial mucosa from these patients demonstrated eosinophilic infiltration, activated by mast cells and Th2 cells. ${ }^{17}$

\section{Cytokine interaction with other cells}

Mast cells are the main source of allergy-related cytokines (IL4, IL-5, IL-6, TNF $\alpha) .{ }^{18}$ Mast cells are equipped with receptors for interaction with cytokines (IL-3R, IL-4R, IL-5R, IL-9R, IL-10R, etc.). ${ }^{19}$ Patients with neutrophilic BA demonstrate significant activity of IL-17. The disease severity correlates with the level of serum IL$17 .{ }^{20} \mathrm{IL}-8$ affects neutrophil function, ${ }^{21}$ while IL-17 attracts neutrophils to the area of inflammation and active infection. ${ }^{22}$ Lymphocytes are divided into three groups according to their ability to produce cytokines: group 1 is the production of IFN $\gamma$, group 2 is the production of IL-5 and IL-13, and group 3 is the production of IL-17 and IL-22. ${ }^{23}$ Due to the involvement of IL-5 in the formation of eosinophilic BA, this cytokine has been called eosinophilic colony-stimulating factor.

IL-17 acts on the respiratory epithelium, stimulating the secretion of a large number of biologically active substances including cytokines IL-4 and IL-5. In addition, IL-17 is an activator of endothelial cells, resulting in the migration of neutrophils to the area of inflammation. ${ }^{24}$ The link between cytokines and cells ensures that they fulfill their main function - the interaction between cells during immunological and other reactions. This link between cytokines and cells can be clearly illustrated by the reaction of the immune system to an antigen stimulus. Through the antigen-presenting cells, the antigen affects the Th0 cells, leading them to differentiate into Th2, with IL-4 participating in this event. Then, with the participation of IL-4, IL-5 and IL-13, there is activation of $\mathrm{B}$ cells, with the formation of specific IgE, which are fixed on the surface of mast cells. When stimulated by the antigen, mast cells secrete histamine, prostaglandins and leukotrienes, which leads to bronchospasm, hypersecretion and mucosal swelling in the respiratory tract. At the same time, exposure to IL-5 causes the Th2 cells to stimulate eosinophils to form leukotrienes and reactive oxygen species. All this, under normal conditions, is aimed at eliminating the antigen, but in patients leads to obstruction and inflammation ${ }^{20}$ 


\section{Anti-cytolkine therapy}

The number of research articles about asthma exceeds 1,500,000 articles, and nearly $6 \%$ of them report on the results of randomized controlled trials for the treatment of BA. However, despite these studies, the treatment options for asthma remain limited, and steroids are the predominant type of medication. ${ }^{25}$ The discovery of different inflammatory phenotypes in patients with BA and the related molecular phenotyping, thanks to new technologies in the field of molecular biology and immunogenetics, have made it possible to synthesize specific monoclonal antibodies, including anti-cytokine antibodies. ${ }^{26}$ Anti-IL monoclonal antibodies should reduce airway inflammation and prevent eosinophilic activation. ${ }^{27}$

Mepolizumab is a humanized monoclonal antibody that blocks IL5 , which moves from the bone marrow and activates eosinophils. ${ }^{28}$ Ortega $\mathrm{HG}$ et $\mathrm{al}^{29}$ used mepolizumab in the treatment of 539 patients with BA. Compared to the placebo, the frequency of disease exacerbations was $47 \%$ lower. The positive effect of treatment with mepolizumab was present with both intravenous and subcutaneous administration in $50 \%$ of patients. Bel EH et al. ${ }^{30}$ treated 135 patients with BA using mepolizumab. The dose of oral glucocorticoids decreased by an average of $50 \%$ in the patient group. There was no decrease in the medication dose in the placebo group. In 2015, the FDA in the United States and the European EMA approved mepolizumab as maintenance therapy in severe eosinophilic BA in adults. ${ }^{31}$

Reslizumab is a humanized monoclonal antibody to IL-5. Castro M et $\mathrm{l}^{32}$ used reslizumab to treat 53 adult patients with severe eosinophilic asthma. There was an improvement in the clinical status, a reduction in the eosinophilic content of the blood and sputum, and an increase in the FEV1. The treatment was most effective in patients with nasal polyps. Benralizumab is a humanized monoclonal antibody to the IL-5 receptor. Castro $\mathrm{M}$ et al ${ }^{32}$ used benralizumab to treat 385 patients with uncontrolled eosinophilic asthma. A single dose of benralizumab reduced the number of serum eosinophils, and reduced the number of exacerbations. Lebrikizumab is a humanized monoclonal antibody that blocks IL-13, which induces periostin secretion by bronchial epithelial cells, ${ }^{33}$ stimulates IgE synthesis, bronchial fibrosis, and respiratory hypersensitivity. ${ }^{34} \mathrm{~J}$ Corren et al..$^{35}$ used lebrikizumab to treat 219 patients with BA. There was an insignificant tendency towards a reduction in the severity of exacerbations as compared to the placebo group. Treatment efficacy was higher in patients with a high serum periostin level. M Noonan et al. ${ }^{36}$ treated 210 patients with BA using lebrikizumab. Treatment efficacy analysis showed no significant changes in the clinical course and FEV1 of BA patients treated with lebrikizumab as compared to the placebo group.

Dupilumab is a humanised monoclonal antibody to the IL-4 receptor, blocking the effects of IL-4 and IL-13. These cytokines have an increased activity in patients with eosinophilic BA and other signs of increased Th2 immune system activity. ${ }^{37}$ Wenzel $\mathrm{S}$ et al. ${ }^{38}$ treated 104 patients with BA using dupilumab, and these patients had fewer exacerbations due to cessation of medications $(p \leq 0.001)$ and an increase in FEV1 $(\mathrm{p} \leq 0.001)$ as compared to the placebo group. Daclizumab is a humanised monoclonal antibody to IL-2, inhibiting cell proliferation and the release of cytokines by cells. Busse WW et al. ${ }^{37}$ used daclizumab to treat 115 adult patients with severe asthma. There was only a small improvement in lung function and control over the disease. Anrucinzumab is a humanised monoclonal antibody to IL13. DeBoever FH et al. ${ }^{39}$ used anrucinzumab to treat 237 patients with severe asthma, who were receiving the maximum dose of ICS. There was a lack of positive change in regards to the eosinophil content and IgE levels. Tralokinumab is a humanized monoclonal antibody to IL-13. May RD et al. ${ }^{40}$ used tralokinumab to treat 194 patients with moderate and severe BA. A moderate improvement in lung function and a reduced use of adrenoceptor agonists was noted.

a. Available publications on the treatment of patients using anticytokine drugs have made the following conclusions.

b. The treatment efficacy of these drugs is low and the clinical effect, if present, does not exceed $50-60 \%$.

c. Individual studies demonstrate a higher effect in the presence of blood or sputum eosinophilia, or increased nitrogen oxide content in exhaled air.

d. There is no information on the iatrogenic effects of these drugs and their contraindications

e. The levels of respective cytokines were not measured before the start and during treatment.

Data analysis of the literature and our own experience suggests that the current knowledge about the level and interaction of CK, and our clinical understanding of this contains a lot of unsolved questions, with the following being the main ones:

A. Is there a difference in the CK levels in different study groups,

B. What combinations of $\mathrm{CK}$, taking into account their levels, are present in different study groups,

C. How statistically significant are the possible links between CK, and if such links exist, how should they be interpreted,

D. What is the role of $\mathrm{CK}$ in sensitisation,

E. Can the results of a study of CK be used for clinical diagnosis and prescription of medications.

Personal data

Study aim: to collect and analyze the facts supporting the involvement of $\mathrm{CK}$ in the pathogenesis, sensitisation and clinical presentation of patients with BA and COPD.

Study objectives:

1. To determine the CK levels in different study groups (during the acute phase of the illness in patients).

2. To determine the frequency (in \%) of different CK combinations, considering their level and numbers.

3. To study the possible $\mathrm{CK}$ combinations, taking their levels into consideration.

4. To analyze the presence and reliability of the links between CK for the entire set of data and for each studied group.

5. To study the involvement of CK in the sensitisation to bacterial and atopic allergens, taking into account the availability and absence of allergic diseases.

\section{Study materials and methods}

The study included 210 persons: group 1 was 32 patients with bronchial asthma light currents (MBA), group 2 was 39 patients with moderate bronchial asthma (MoBA), group 3 was 39 patients 
with moderate bronchial asthma combined with chronic obstructive pulmonary disease (MoBA+COPD), group 4 was 38 patients with chronic obstructive pulmonary disease (COPD), group 5 was 17 patients with community-acquired pneumonia (CAP), group 6 was 25 patients with HT and IHD, and group 7 consisted of 20 healthy subjects. The age and sex of the subjects are shown in Table 1.

General clinical, laboratory and instrumental studies were carried out. Serum allergen-specific IgE antibodies and cytokines were determined using solid phase immunosorbent assays. The presence of IgE to tick and house dust allergens, as well as to grass pollen, tree, weed and flower allergens, $S$. pneumon, $H$. influenzae, and $N$. rerflava was determined. For the overall assessment of infectious sensitisation, the infectious potential (INFP) was determined, and for the overall assessment of atopic sensitisation, the atopic potential (ATP) was determined for each subject. Infectious potential: IgE to Strept. pneumon. + IgE to Haemoph. influenzae + IgE to Neiss. perflavae (KE/l).Atopic potential: $\operatorname{IgE}$ to tick $+\operatorname{IgE}$ to dust $+\operatorname{IgE}$ to field grasses $+\operatorname{IgE}$ to tree pollen $+\operatorname{IgE}$ to weeds $+\operatorname{IgE}$ to flowers (classes). To assess the severity of each indicator, it was assigned a level in each subject: low, medium and high. The levels were calculated when analyzing the histograms of each indicator. The level boundaries of the studied indicators are shown in Table 2. Statistical 6.1 by Stat Soft, Inc. was used for the statistical data analysis. When interpreting the results, 0.05 was the critical value of the significance level. The mean and standard deviation $(\mathrm{M} \pm \mathrm{s})$ were used as descriptive statistics for the quantitative signs. The Student's t-test and Pearson correlation coefficient were also used.

Table I Patient characteristics according to age and sex

\begin{tabular}{|c|c|c|c|c|c|}
\hline No & Study groups & $\mathbf{N}$ & Average age $\mathrm{M} \pm \mathrm{s}$ & Men \% & $\begin{array}{l}\text { Women } \\
\%\end{array}$ \\
\hline I & MBA & 32 & $32.75 \pm 11.753$ & 25 & 75 \\
\hline 2 & MoBA & 39 & $45.38 \pm 18.104$ & 25.6 & 74.4 \\
\hline 3 & MoBA+COPD & 39 & $60.85 \pm 10.835$ & 56.4 & 43.6 \\
\hline 4 & COPD & 38 & $64.45 \pm 8.096$ & 78.9 & 21.1 \\
\hline 5 & CAP & 17 & $44.88 \pm 17.345$ & 52.9 & 47.1 \\
\hline 6 & HT, IHD & 25 & $59.68 \pm 18.314$ & 32 & 68 \\
\hline 7 & Healthy & 20 & $35.15 \pm 11.726$ & 25 & 75 \\
\hline
\end{tabular}

Table 2 The level boundaries of the studied indicators using the entire data sum

\begin{tabular}{|c|c|c|c|c|c|c|c|c|c|c|}
\hline \multirow{2}{*}{ Indicator } & \multirow{2}{*}{$\mathbf{N}$} & \multicolumn{3}{|c|}{ Low level } & \multicolumn{3}{|c|}{ Medium level } & \multicolumn{3}{|c|}{ High level } \\
\hline & & $\mathbf{n}$ & $\%$ & level boundary & $\mathbf{n}$ & $\%$ & level boundary & $\mathbf{n}$ & $\%$ & level boundary \\
\hline IL-4 & 133 & 49 & 36.8 & $\leq 1.6$ & 44 & 33.1 & ]1.6-2.6] & 40 & 30.1 & $>2.6$ \\
\hline IL-6 & 90 & 32 & 35.6 & $\leq 1.7$ & 29 & 32.2 & ]1.7-3.7] & 29 & 32.2 & $>3.7$ \\
\hline IL- I0 & 134 & 47 & 35.1 & $\leq 3.7$ & 44 & 32.8 & ]3.7-7.I] & 43 & 32.1 & $>7.1$ \\
\hline IL-I 7 & 148 & 51 & 34.5 & $\leq 2.5$ & 49 & 33.1 & ]2.5-4.2] & 48 & 32.4 & $>4.2$ \\
\hline $\mathrm{IFN} \gamma$ & 133 & 44 & 33.1 & $\leq 4.7$ & 44 & 33.1 & ]4.7-8.5] & 45 & 33.8 & $>8.5$ \\
\hline $\mathrm{TNF} \alpha$ & 177 & 59 & 33.3 & $\leq 2.5$ & 57 & 32.2 & ]2.5-4.4] & 61 & 34.5 & $>4.4$ \\
\hline INFP & 165 & 55 & 33.3 & $\leq 72$ & 55 & 33.3 & ]72-I55] & 55 & 33.3 & $>155$ \\
\hline ATP & 188 & 62 & 33 & $\leq 4$ & 62 & 33 & $14-7$ & 64 & 34 & $>7$ \\
\hline
\end{tabular}

\section{Results and discussion}

The CK levels in all of the groups are shown in Table 3. There were different CK levels in the study subjects of all the groups: the incidence range of the low level varied from $4.3 \%$ to $61.5 \%$, while the incidence range of the high level varied from $11.8 \%$ to $75.0 \%$ of the subjects. Different CK levels were present in all of the groups, including patients with ischaemic heart disease, HT, and healthy volunteers. The incidence (\%) of a combination of several cytokines of the same level, in each group, is presented in Figure 1. The " 0 " frequency means that a particular group lacks CK at this level. In all the groups, the frequency of a combination of several $\mathrm{CK}$ of one level did not differ significantly. Table 4 presents the incidence of a combination of several high-level CK in one study subjects. 3.1$39.1 \%$ of subjects had high levels of several CK, irrespective of the study group, including patients with HT, IHD, and healthy volunteers. The incidence of a number of high-level CK is presented in Table
4. High-level CK were absent in $19.4-51.6 \%$ of all subjects, while $16.1-27.8 \%$ of patients had one high-level CK. Combinations of different amounts of high-level CK were noted in all of the study groups.A 9-cell matrix (Table 5) was used to study the presence of combinations of different levels of two CK, which contained the fixed percentage of the nine combinations of two CK in the study group. For example, the matrix shown in Figure 2 was designed to identify if any group had one of the nine combinations of the levels of IL-4 and IL-10. The first cell contained the percentage of subjects in a group with low levels of IL-4 and IL-10, while the ninth cell contained the percentage of the subjects in a group with high levels of IL-4 and IL10. The remaining 7 cells contained percentages of subjects with the corresponding levels of IL-4 and IL-10. Figure 2 shows a graphical demonstration of IL-4 and IL-10 combinations in the subjects from different groups. Thus, the incidence was obtained for each of the 9 combination options of two levels of comparable CK for each study group. In order to determine whether the frequency range of the levels 
of two comparable CK, obtained from the 9-cell matrix, is different in different study groups, and not having the opportunity to include the whole actual material in this article, we present the frequency range (minimum and maximum frequency in \%) of the comparable CK for each of the study groups (Table 6). The data obtained indicate that the frequency range of values of the investigated CK does not significantly differ between the different study groups, including patients and healthy volunteers.

For physiological and clinical interpretation of the multiple links between the studied cytokines, we need to know whether there is a statistically significant link between them. The statistical links between pairs of cytokines were investigated using the whole data, and for each group of subjects. The results of the research into links across the whole data are shown in Table 7. The interaction of IL-4 with IL-10, IL-17, interferon-gamma; IL-6 with TNF $\alpha$; IL-10 with IL17 and interferon gamma; and IL-17 with interferon gamma and TNF $\alpha$ have a statistically significant link. The function of these significant links between CK is unknown, but undoubtedly, these links are relevant to the functioning of CK both in healthy and disease states. The results of the study into the links between CK in the different study groups are presented in Table 8 . In $25 \%$ of comparable CK pairs, the interaction between them was not statistically significant. To assess the involvement of cytokines in the sensitisation to bacterial and atopic allergens, a statistical analysis was carried out of the comparable indicators characterizing infectious (INFP) and atopic (ATP) sensitisation, with different levels of each of the studied CK in patients without allergic diseases (COPD $+\mathrm{CAP}+\mathrm{HT}$ and IHD + HEALTHY) versus patients with allergic diseases (MBA + MoBA + MoBa combined with COPD) (Table $9 \&$ Figure $3 \&$ Figure 4). It was found that: 1) there was a significant difference between INFP in subjects without allergy and subjects with allergy, in the presence of predominantly high levels of CK (apart from TNF $\alpha$ ); 2) there was no significant difference found in the indicator characterizing ATP in the study groups, in any of the CK levels (apart from high level of IL-4); 3) there was no significant link established between TNF $\alpha$ and different INFP and ATP in the study subjects; 4) higher levels of INFP and ATP in the study subjects without allergies again confirms the earlier hypothesis that in people without allergies, specific $\operatorname{IgE}$ antibodies to infectious and non-infectious allergens have a protective role. $^{41}$

Table 3 Frequency (in \%) of the CK levels in the study groups

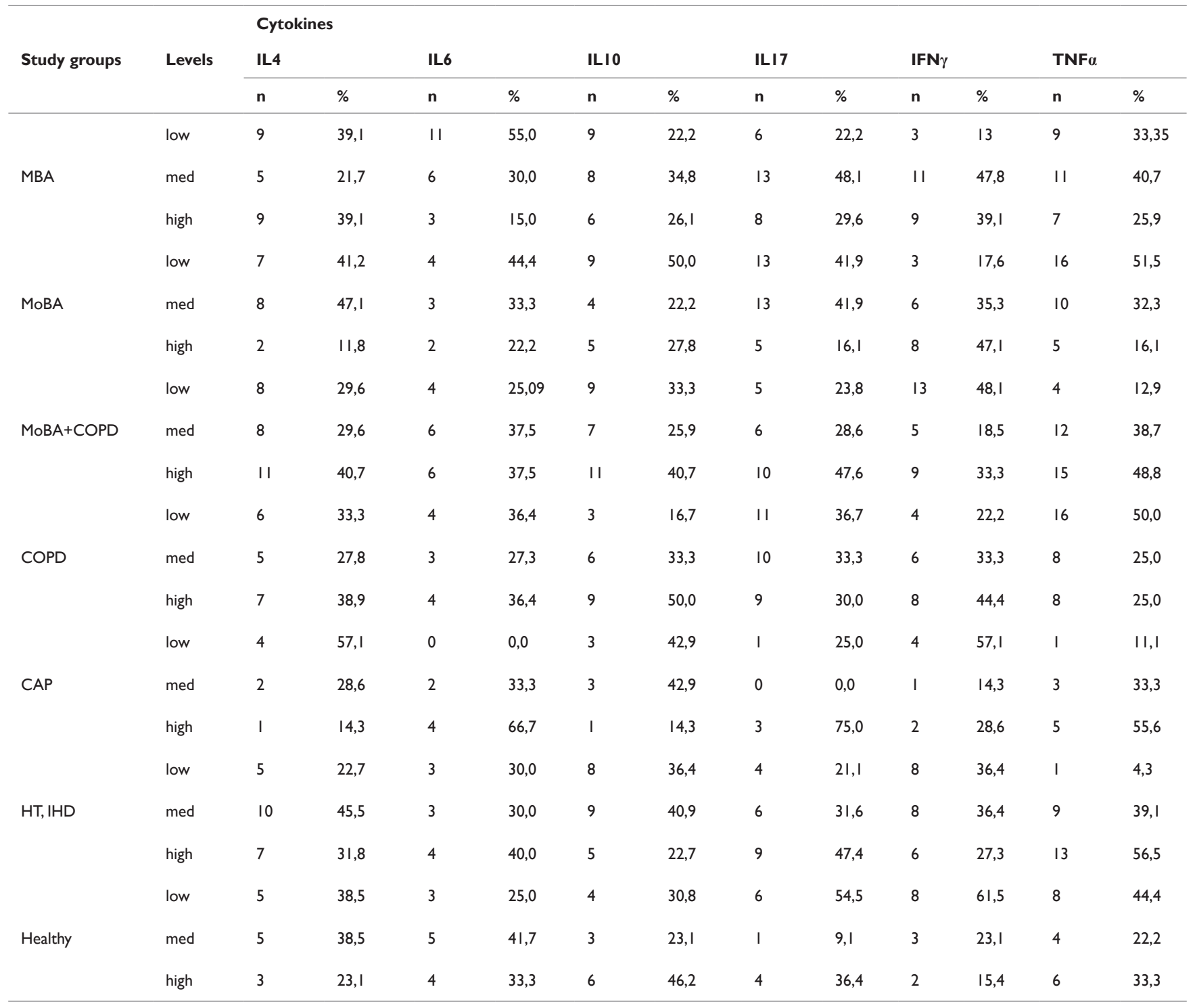



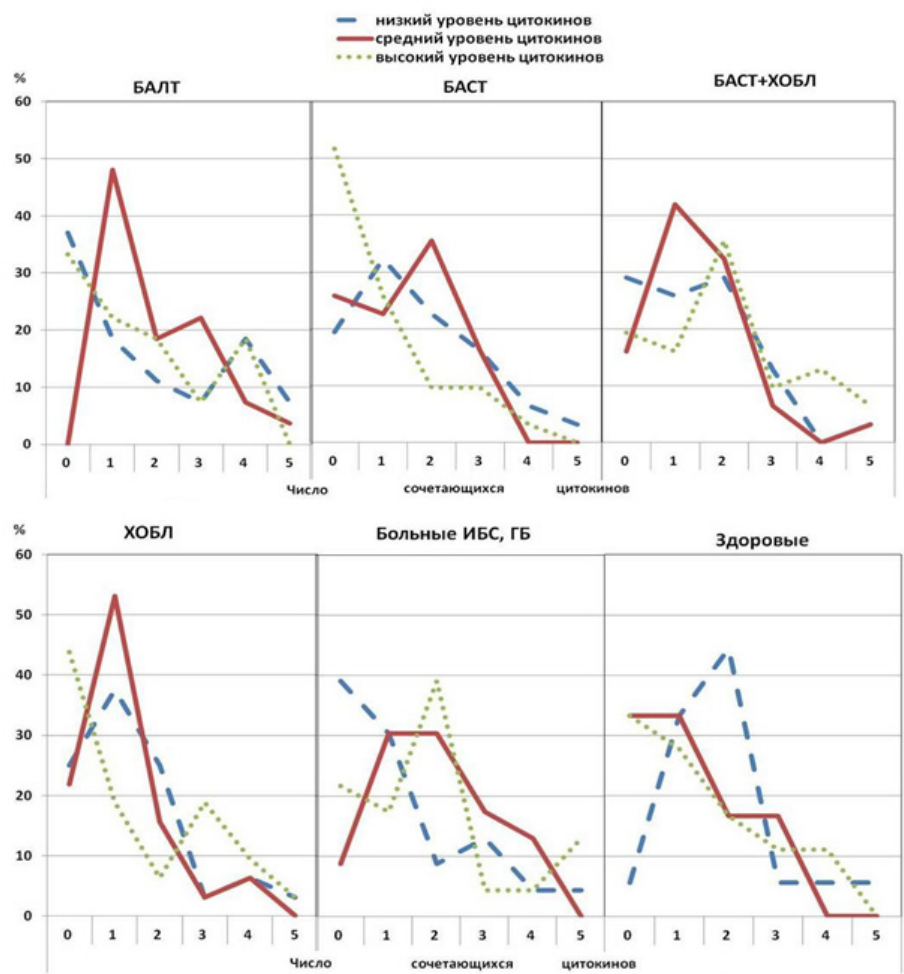

Figure I Incidence (\%) of the combined five CK, considering the number and level of CK.

Table 4 The incidence (in \%) of a different number of high-level cytokines in one study subject

Study groups

The number of high-level CK in one subject

\begin{tabular}{|c|c|c|c|c|c|c|c|}
\hline & \multirow[t]{2}{*}{$\mathbf{N}$} & 0 & I & 2 & 3 & 4 & 5 \\
\hline & & \multicolumn{6}{|c|}{ the frequency percentage of this number of high-level CK } \\
\hline MBA & 27 & 33,3 & 22,2 & 18,5 & 7,4 & 18,5 & 0,0 \\
\hline MoBA & 31 & 51,6 & 25,8 & 9,7 & 9,7 & 3,2 & 0,0 \\
\hline MoBA+COPD & 31 & 19,4 & 16,1 & 35,5 & 9,7 & 12,9 & 6,5 \\
\hline COPD & 32 & 43,8 & 18,8 & 6,3 & 18,8 & 9,4 & 3,1 \\
\hline IHD, HT & 23 & 21,7 & 17,4 & 39,1 & 4,3 & 4,3 & 13,0 \\
\hline Healthy & 18 & 33,3 & 27,8 & 16,7 & $\mid \mathrm{I}, \mathrm{I}$ & $\mid \mathrm{I}, \mathrm{I}$ & 8,0 \\
\hline
\end{tabular}

Table 5 The matrix to determine the frequency of the nine combinations of two CK, considering their level (low, medium, high) in \%

\begin{tabular}{lllll}
\hline Which cytokines & & IL4 & & High level \\
\cline { 3 - 5 } & Low level & Medium level & 3 \\
\hline ILI0 & low level & 2 & 6 \\
medium level & 4 & 5 & 9 \\
high level & 7 & 8 &
\end{tabular}




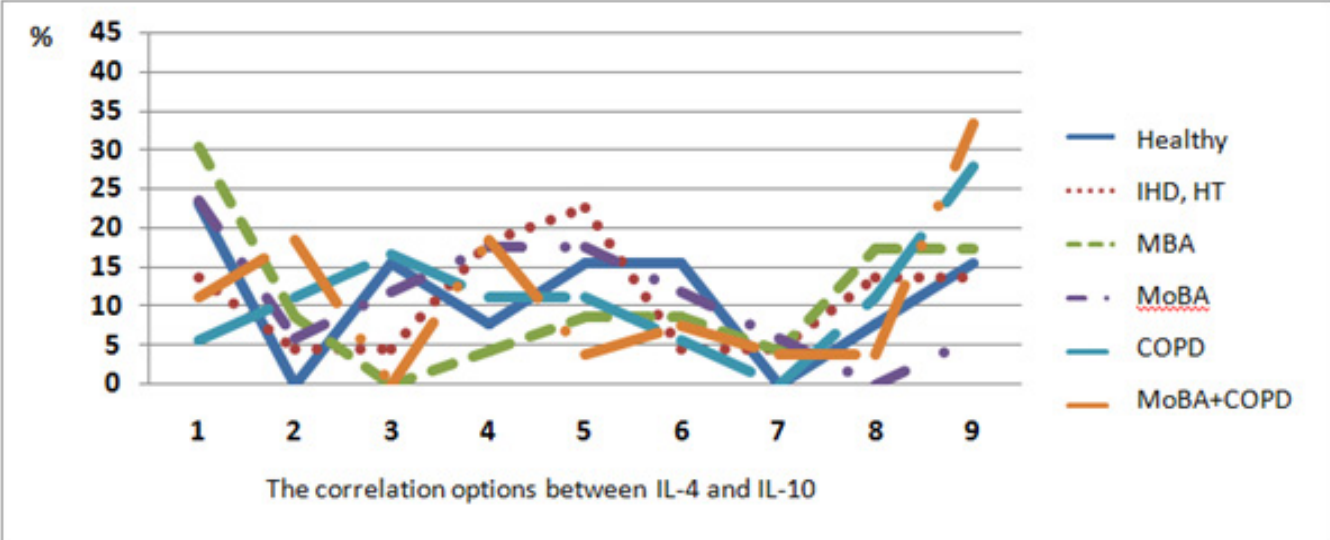

Figure 2 The distribution of subjects in \%\%, depending on the ratio of IL4 and ILIO.

Table 6 The frequency range (in \%) of the combinations of different CK levels in the study groups

\begin{tabular}{|c|c|c|c|c|c|c|c|c|c|c|}
\hline Study groups & IL4 \& IL6 & IL4 \& ILII0 & IL4 \& ILI7 & IL4 \& IFN $\gamma$ & IL4 \& TNF $\alpha$ & IL6 \& ILI0 & IL6 \& ILI7 & IL6 \& IFN $\gamma$ & IL-10 \& IL-17 & IL-I0 \& GIN \\
\hline MBA & $0,0-30,0$ & $0,0-30,4$ & $0,0-21,7$ & $0,0-21,7$ & $4,3-21,7$ & $0,0-35,0$ & $0,0-20,0$ & $0,0-20,0$ & $0,0-21,7$ & $0,0-26,1$ \\
\hline MoBA & $0,0-33,0$ & $0,0-23,5$ & $0,0-17,6$ & $0,0-23,5$ & $0,0-23,5$ & $0,0-33,3$ & $0,0-22,2$ & $0,0-33,0$ & $0,0-33,7$ & $0,0-29,4$ \\
\hline MoBA + COPD & $0,0-25,1$ & $0,0-33,0$ & $0,0-41,2$ & $0,0-29,6$ & $0,0-25,9$ & $0,0-25,0$ & $0,0-33,3$ & $0,0-37,5$ & $0,0-50,0$ & $0,0-38,9$ \\
\hline COPD & $0,0-27,3$ & $0,0-27,8$ & $0,0-25,0$ & $5,6-16,7$ & $0,0-22.2$ & $0,0-27,3$ & $0,0-22,2$ & $0,0-27,3$ & $0,0-50,0$ & $0,0-38,9$ \\
\hline CAP & $0,0-33,3$ & $0,0-42,9$ & $0,0-50,0$ & $0,0-42,9$ & $0,0-42,9$ & $0,0-33,3$ & $0,0-56,7$ & $0,0-33,3$ & $0,0-50,0$ & $0,0-42,9$ \\
\hline $\mathrm{HT}, \mathrm{IHD}$ & $0,0-40,0$ & $4,5-18,2$ & $0,0-50,0$ & $0,0-27,3$ & $0,0-27,3$ & $0,0-30,0$ & $0,0-50,0$ & $0,0-18,2$ & $0,0-27,8$ & $0,0-18,2$ \\
\hline Healthy & $0,0-16,7$ & $0,0-15,4$ & $0,0-22,2$ & $0,0-38,5$ & $0,0-30,8$ & $0,0-25,0$ & $0,0-50,0$ & $0,0-30,8$ & $0,0-33,3$ & $0,0-30,8$ \\
\hline
\end{tabular}

Table 7 Significant links between CK, the overall data sum irrespective of the study groups

\begin{tabular}{llll}
\hline Comparable CK & N & R & P \\
\hline IL4 \& ILI0 & 98 & 0,44 & 0,000006 \\
IL4 \& ILI7 & 80 & 0,44 & 0,000039 \\
IL4 \& IFN $\gamma$ & 98 & 0,50 & 0,000000 \\
IL6 \& TNF $\alpha$ & 7 II & 0,38 & 0,000932 \\
ILI0 \& ILI7 & 81 & 0,65 & 0,000000 \\
ILI0 \& IFN $\gamma$ & 98 & 0,53 & 0,000000 \\
ILI7 \& IFN $\gamma$ & 80 & 0,56 & 0,000000 \\
ILI7 \& TNF $\alpha$ & II8 & 0,40 & 0,000007
\end{tabular}

Table 8 Significant links between CK in the study groups (p)

\begin{tabular}{llllllllllllllll}
\hline \multirow{2}{*}{ Study groups } & IL4 \& & $\begin{array}{l}\text { IL4 \& } \\
\text { ILI0 }\end{array}$ & $\begin{array}{l}\text { IL4 \& } \\
\text { ILI7 }\end{array}$ & $\begin{array}{l}\text { IL4 \& } \\
\text { IFN } \gamma\end{array}$ & $\begin{array}{l}\text { IL4 \& } \\
\text { TNF } \alpha\end{array}$ & $\begin{array}{l}\text { IL6 \& } \\
\text { ILI0 }\end{array}$ & $\begin{array}{l}\text { IL6 \& } \\
\text { ILI7 }\end{array}$ & $\begin{array}{l}\text { IL6 \& } \\
\text { IFN } \gamma\end{array}$ & $\begin{array}{l}\text { IL6 \& } \\
\text { TNF } \alpha\end{array}$ & $\begin{array}{l}\text { IL4I0 \& } \\
\text { ILI7 }\end{array}$ & $\begin{array}{l}\text { ILI0 \& } \\
\text { IFN } \gamma\end{array}$ & $\begin{array}{l}\text { ILI0 \& } \\
\text { TNF } \alpha\end{array}$ & $\begin{array}{l}\text { ILI7 \& } \\
\text { IFN } \gamma\end{array}$ & $\begin{array}{l}\text { ILI7 \& } \\
\text { TNF } \alpha\end{array}$ & $\begin{array}{l}\text { IFN } \gamma \text { \& } \\
\text { TNF } \alpha\end{array}$ \\
\hline MBA & 0,045 & 0,008 & 0,015 & 0,083 & 0,559 & 0,605 & 0,845 & 0,930 & 0,654 & 0,001 & 0,000 & 0,191 & 0,002 & 0,192 & 0,059 \\
MoBA & 0,776 & 0,458 & 0,691 & 0,891 & 0,305 & 0,907 & 0,571 & 0,081 & 0,275 & 0,119 & 0,464 & 0,106 & 0,895 & 0,135 & 0,966 \\
MoBA+COPD & 0,038 & 0,002 & 0,007 & 0,000 & 0,020 & 0,387 & 0,658 & 0,330 & 0,196 & 0,002 & 0,000 & 0,585 & 0,004 & 0,020 & 0,202 \\
COPD & 0,285 & 0,340 & 0,209 & 0,237 & 0,229 & 0,830 & 0,965 & 0,872 & 0,112 & 0,000 & 0,006 & 0,860 & 0,005 & 0,019 & 0,514 \\
CAP & 0,913 & 0,288 & - & 0,067 & 0,175 & 0,110 & - & 0,026 & 0,704 & - & 0,006 & 0,052 & - & 0,800 & 0,071 \\
HT, IHD & 0,188 & 0,151 & 0,160 & 0,105 & 0,654 & 0,234 & 0,0136 & 0,325 & 0,117 & 0,041 & 0,020 & 0,816 & 0,253 & 0,045 & 0,716 \\
Healthy & 0,399 & 0,7878 & 0,083 & 0,657 & 0,639 & 0,335 & 0,8268 & 0,018 & 0,006 & 0,004 & 0,521 & 0,528 & 0,354 & 0,698 & $-0,02$ \\
\hline
\end{tabular}


Table 9 Comparison of INFP and ATP with different CK levels in patients without allergic diseases and in patients with allergic diseases

\begin{tabular}{|c|c|c|c|c|c|c|c|c|c|c|c|}
\hline \multirow[t]{2}{*}{ IL } & & & \multicolumn{4}{|c|}{ Without allergic diseases } & \multicolumn{4}{|c|}{ With allergic diseases } & \multirow[t]{2}{*}{$\mathbf{P}$} \\
\hline & & & $\mathbf{N}$ & Mediana & $25 \%$ & $75 \%$ & $\mathbf{N}$ & Mediana & $25 \%$ & $75 \%$ & \\
\hline \multirow[t]{6}{*}{ IL4 } & $\mathrm{L}$ & INFP & 19 & 99.84 & 58.3 & 132.22 & 28 & 66.035 & 35.255 & 92.6 & 0.080926 \\
\hline & & ATP & 20 & 6.5 & 4 & 9 & 29 & 5 & 3 & 7 & 0.106288 \\
\hline & M & INFP & 19 & 156.42 & 82.63 & 281 & 15 & 68.4 & 45.48 & 92.45 & 0.003075 \\
\hline & & ATP & 22 & 6 & 5 & 8 & 22 & 7.5 & 4 & 9 & 0.568834 \\
\hline & $\mathrm{H}$ & INFP & 11 & 154.54 & 98.06 & 259.67 & 12 & 58.87 & 36.235 & 116.04 & 0.043879 \\
\hline & & ATP & 18 & 9 & 7 & 10 & 22 & 6 & 4 & 8 & 0.014925 \\
\hline \multirow[t]{6}{*}{ IL6 } & $\mathrm{L}$ & INFP & 10 & 75.52 & 43.21 & 103.84 & 20 & 49.93 & 20.39 & 81.35 & 0.130729 \\
\hline & & ATP & 10 & 8 & 6 & 12 & 22 & 6.5 & 4 & 9 & 0.091868 \\
\hline & $M$ & INFP & 11 & 121.01 & 98.06 & 281.34 & 13 & 75.5 & 52.17 & 92.45 & 0.08213 \\
\hline & & ATP & 13 & 6 & 4 & 7 & 16 & 5 & 3 & 8 & 0.681573 \\
\hline & $\mathrm{H}$ & INFP & 16 & 136.48 & 88.38 & 269.97 & 13 & 82.44 & 58.3 & 86.81 & 0.055535 \\
\hline & & ATP & 16 & 5.5 & 4.5 & 8 & 13 & 5 & 3 & 6 & 0.249468 \\
\hline \multirow[t]{6}{*}{ ILIO } & $\mathrm{L}$ & INFP & 17 & 103.84 & 43.21 & 156.42 & 27 & 67.02 & 45.48 & 90.86 & $0.17|28|$ \\
\hline & & ATP & 18 & 6 & 5 & 9 & 29 & 5 & 4 & 7 & 0.272104 \\
\hline & $M$ & INFP & 17 & 143.4 & 76.91 & 281 & 20 & 63.28 & 33.69 & 112.29 & $0.04847 \mid$ \\
\hline & & ATP & 21 & 8 & 4 & 9 & 23 & 6 & 3 & 9 & 0.455949 \\
\hline & $\mathrm{H}$ & INFP & 15 & 121.01 & 99.84 & 258.93 & 9 & 92.45 & 48.78 & 94.3 & 0.00435 \\
\hline & & ATP & 21 & 8 & 6 & 10 & 22 & 7.5 & 5 & 8 & 0.163998 \\
\hline \multirow[t]{6}{*}{ ILI7 } & $\mathrm{L}$ & INFP & 21 & 88.79 & 72.48 & 130.82 & 28 & 93.6 & 57.46 & 205.01 & 0.77164 \\
\hline & & ATP & 22 & 5 & 3 & 8 & 29 & 5 & 4 & 8 & 0.813742 \\
\hline & $M$ & INFP & 16 & 176.25 & 93.95 & 265.95 & 25 & 61.5 & 37.1 & 246.88 & 0.051924 \\
\hline & & ATP & 17 & 8 & 6 & 9 & 32 & 7 & 4 & 9 & 0.229724 \\
\hline & $\mathrm{H}$ & INFP & 16 & 118.93 & 78.18 & 243.02 & 12 & 71.95 & 50.48 & 94.32 & 0.02918 \\
\hline & & ATP & 25 & 8 & 6 & 10 & 23 & 6 & 4 & 9 & 0.06908 \\
\hline \multirow[t]{6}{*}{$\mathrm{IFN} \gamma$} & $\mathrm{L}$ & INFP & 21 & 143.4 & 82.63 & 281 & 17 & 78.44 & 53.97 & 90.86 & 0.041813 \\
\hline & & ATP & 24 & 5 & 2 & 9.5 & 20 & 4.5 & 2.5 & 6 & 0.461678 \\
\hline & $M$ & INFP & 16 & 96.32 & 23.64 & 222.05 & 23 & 61.5 & 32 & 82.45 & 0.107292 \\
\hline & & ATP & 18 & 8 & 6 & 9 & 26 & 6.5 & 4 & 9 & 0.092604 \\
\hline & $\mathrm{H}$ & INFP & 12 & 130.88 & 106.08 & 224.31 & 15 & 65.05 & 24.5 & 96.55 & 0.010208 \\
\hline & & ATP & 18 & 8.5 & 7 & 10 & 27 & 7 & 4 & 9 & 0.130115 \\
\hline \multirow[t]{6}{*}{$\mathrm{TNF} \alpha$} & $\mathrm{L}$ & INFP & 23 & 109.95 & 76.12 & 180.24 & 32 & 80.21 & 34.14 & 220.57 & 0.51434 \\
\hline & & ATP & 26 & 6.5 & 3 & 9 & 33 & 5 & 4 & 8 & 0.52916 \\
\hline & $M$ & INFP & 21 & 123.06 & 82.63 & 259.67 & 22 & 85.72 & 47.73 & 167.47 & 0.112125 \\
\hline & & ATP & 24 & 8 & 5.5 & 9 & 33 & 6 & 4 & 8 & 0.115587 \\
\hline & $\mathrm{H}$ & INFP & 27 & 188.95 & 95.14 & 286.48 & 22 & 83.76 & 61.5 & 175.7 & 0.115184 \\
\hline & & ATP & 32 & 7 & 4 & 9 & 29 & 6 & 3 & 8 & 0.459783 \\
\hline
\end{tabular}




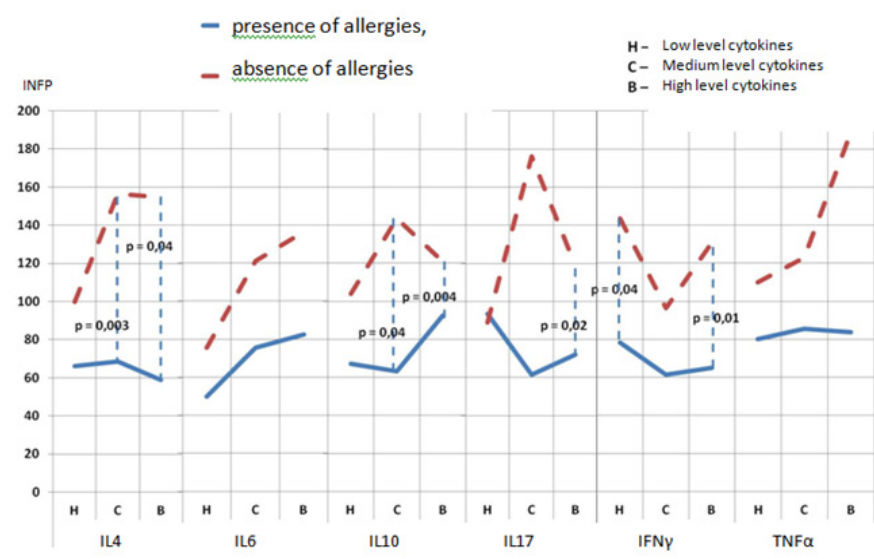

Figure 3 INFP in the presence and absence of clinically significant allergies, taking into consideration the CK level.

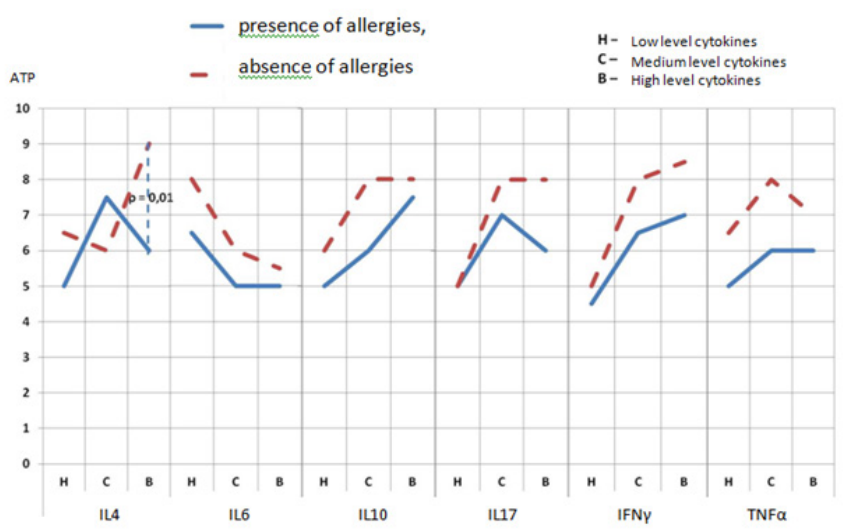

Figure 4 ATP in the presence and absence of clinically significant allergies, taking into consideration the CK level.

\section{Conclusion}

A. There is a wide range of CK levels in all of the study subjects, including the comparison group (HT and IHD) and the healthy control group.

B. In all of the study subjects, the frequency of combinations of several CK of one level did not differ significantly. There were high levels of several CK in 3.1-39.1\% of subjects, irrespective of the study group, including patients with HT, IHD, and healthy volunteers.

C. The frequency range of combinations of different CK levels did not significantly differ between the groups, including patients with HT, IHD, and healthy volunteers.

D. There was a statistically significant link between cytokines found across all the study subjects. Significant links between cytokines in specific groups were noted in approximately $25 \%$ of comparable CK pairs for each group of patients.

E. There was a significant difference between the severity of INFP in patients without allergies and the severity of INFP in patients with allergies, in the presence of predominantly high levels of cytokines (except TNF), which can be considered as another pathogenesis mechanism of sensitization and allergy.
F. The results of the study of CK levels and CK combinations, the so-called cytokine profile, demonstrate that they cannot be used for clinical diagnosis, including nosological diagnosis, assessment of disease severity, and for selecting individual therapy, including anti-cytokine medications.

\section{Acknowledgements}

None.

\section{Conflicts of interest}

The authors declare that there is no conflict of interest.

\section{References}

1. Fedoseev GB, Korovina OV, Tenigina NG. The comprehensive diagnosis of different clinical and pathogenetic types of bronchial asthma. Therapy Archive. 1977;6:51-55.

2. Adcock IM, Lane SJ. Corticosteroid-insensitive asthma: molecular mechanisms. J Endocrinol. 2003;178(3):347-355.

3. Icuhara $\mathrm{K}$, Matsumoto $\mathrm{H}$, Ohta $\mathrm{S}$, et al. Recent developments regarding periostin in bronchial asthma. Allergol Int. 2015;64 (Suppl):s3-s10.

4. Desai M, Oppenheimer J. Elucidating asthma phenotypes and endotypes: progress towards personalized medicine. Annals Allergy Asthma Immunol. 2016;116(5):394-401

5. Wenzel SE. Asthma phenotypes: evolution from clinical to molecular approaches. Nat Med. 2012;18(5):716-725.

6. Muraro A, Lemanske RF, Hellings PW, et al. Precision medicine in patients with allergic diseases: Airway diseases and atopic dermatitis - PRACTALL documents of the European Academy of Allergy and Clinical Immunology and the American Academy of Allergy, Asthma and Immunology. J Allergy Clin Immumology. 2016;137(5):1347-1358.

7. Simpson JL, Scott R, Boyle MJ, et al. Inflammatory subtypes in asthma: assessment and identification using induced sputum. Respirology. 2006;1(1):54-61.

8. Fedoseev GB, Trofimov VI, Negrutsa KV, et al. Sputum characteristics for the assessment of the presence and nature of inflammation in the bronchopulmonary tree in patients with bronchial asthma and chronic obstructive pulmonary disease. Russian Journal of Allergy. 2015;1:15-27.

9. Zissler UM, Essen-von Bieren J, Jakwerth CA, et al. Current and future biomarkers in allergic asthma. J Allergy. 2016;71(4):475-494.

10. Hamid QA, Minshall EM. Molecular pathology of allergic disease. $J$ Allergy Clin Immunol. 2000;105(1):20-36.

11. Moldoveanu B, Otmishi P, Jani P, et al. Inflammatory mechanisms in lung. Inflamm Res. 2009;2:1-11.

12. Commins SP, Borish L, Steinke JW. Immunologic messenger molecules: Cytokines, interferons and chemokines. $J$ Allergy Clin Immunol. 2010;125(2):S53-S72.

13. Zimmermann N, Hershey GK, Foster PS, et al. Chemokines in asthma: Cooperative interaction between chemokines and IL-13. J Allergy Clin Immunol. 2003;111(2):227-242.

14. Barnes PJ. The cytokine network in asthma and chronic obstructive pulmonary disease. J Clin Invest. 2008;118(11):3546-3556.

15. Uhm TG, Kim BS, Chung IY. Eosinophil development, regulation of eosinophil-specific genes, and role of eosinophils in pathogenesis of asthma. Allergy Asthma Immunol Res. 2012;4(2):68-79. 
16. Varricchi G, Bagnasco D, Borriello F, et al. Interleukin-5 pathway inhibition in the treatment of eosinophilic respiratory disorders: evidence and unmet needs. Curr Opin Allergy Clin Immunol. 2016;16(2):186-200.

17. Gao P, Simpson JL, Zhang J, et al. Galection-3: its role in asthma and potential as an anti-inflammatory target. Respir Res. 2013;14:136-145.

18. Bradding $\mathrm{P}$, Feather $\mathrm{IH}$, Howarth $\mathrm{PH}$, et al. Interleukin 4 is localized to and released by human mast cells. J Exp Med. 1992;176(5):1381-1386.

19. Stone KD, Prussin C, Metcalfe DD. IgE, mast cells, basophils and eosinophils. J Allergy Clin Immunol. 2010;125(2 Suppl 2):S73-S80.

20. Trevor JL, Deshane JS. Refractory asthma: mechanisms, targets, and therapy. J Allergy. 2014;69(7):817-827.

21. Pelletier M, Maggi L, Micheletti A, et al. Evidence for a crosstalk between human neutrophils and Th17 cells. Blood. 2010;115(2):335-343.

22. Cua DJ, Tato CM. Innate IL-17-producing cells: the sentinels of the immune system. Nat Rev Immunol. 2010;10(7):476-489.

23. Spits S, Artis D, Colonna M, et al. Innate lymphoid cells a proposal for uniform nomenclature. Nat Rev Immunol. 2013;13(2):145-149.

24. Roussel L, Houle F, Chan C, et al. IL-17 promotes p38 MARK-dependent endothelial activation enhancing neutrophil recruitment to sites of inflammation. J Immunol. 2010;184(8):4531-4537.

25. Carr TF, Kraft M. Update in asthma 2014. Am J Respir Crit Care Med. 2015;192(2):157-163.

26. Manzella F, Lusuardi M, Galeone C, et al. Tailored therapy for severe asthma. Multidiscip Respir Med. 2015;10(1):1-5.

27. Castro M, Mathur S, Hargreave F, et al. Reslizumab for poorly controlled eosinophilic asthma: a randomized, placebo-controlled study. Am J Respir Crit Care Med. 2011;184(10):1125-1132.

28. Walsh GM. An update on emerging drags for asthma. Expert Opin Emerg Drags. 2012;17(1):37-42.

29. Ortega HG, Liu MC, Pavord ID, et al. Mepolizumab treatment in patients with severe eosinophilic asthma. New Engl J Med. 2014;371(13):11981207.

30. Bel EH, Wenzel SE, Thompson PJ, et al. Oral glucocorticoid-sparing effect of mepolizumab in eosinophilic asthma. New Engl J Med. 2014;371(13):1189-1197.
31. Varricchi G, Begnasco D, Borriello F, et al. Interleucin-5 pathway inhibition in the treatment of eosinophilic respiratory disorders: evidence and unmet needs. Curr Opin Allergy Clin Immunol. 2016;16(2):186-200.

32. Castro M, Wenzel SE, Bleecker ER, et al. Benralizumab, an antiinterleukin 5 receptor alpha monoclonal antibody, versus placebo for uncontrolled eosinophilic asthma: a phase $2 \mathrm{~b}$ randomized dose-ranging study. Lancet Respir Med. 2014;2(11):879-890.

33. Sidhu SS, Yuan S, Innes AL, et al. Roles of epithelial cell-deriostin periostin in TGF-beta activation, collagen production, and collagen gel elasticity in asthma. Proc Natl Acad Sci USA. 2010;107(32):14170-14175.

34. Humbert M, Durham SR, Kimmitt P, et al. Elevated expression of messenger ribonucleic acid encoding IL-13 in the bronchial mucosa of atopic and nonatopic subjects with asthma. J Allergy Clin Immunol. 1997;99(5):657-665.

35. Corren J, Busse W, Meltzer EO, et al. A randomized, controllel, phase 2 study of AMG 317, an IL-4Ralpha antagonist, in patients with asthma. Am J Respir Crit Care Med. 2010;181(8):788-796.

36. Noonan M, Korenblat P, Mosesona S, et al. Dose-ranging study of lebrikizumab in asthmatic patients not receiving inhaled steroids. $J$ Allergy Clin Immunol. 2013;132(3):567-574.

37. Busse WW, Israel E, Nelson HS, et al. Daclizumab improves asthma control in patients with moderate to severe persistent asthma: a randomized, controlled trial. Am J Respir Crit Care Med. 2008;178(10):1002-1008.

38. Wenzel S, Ford L, Pearlman D, et al. Dupilumab in persistant asthma with elevated eosinophil levels. New Engl J Med. 2013;368(26):2455-2466.

39. De Boever EH, Ashman C, Cahn AP, et al. Efficacy and safety of an antiIL-13 mAb in patients with severe asthma: a randomized trial. J Allergy Clin Immunol. 2014;133(4):989-996.

40. May RD, Monk PD, Cohen ES, et al. Preclinical development of CAT-354, an IL-13 neutralizing antibody, for the treatment of severe uncontrolled asthma. Br J Pharmacol. 2012;166(1):177-193.

41. Fedoseev GB, Trofimov VI, Timchik VG, et al. Infectious and noninfectious sensitisation in patients with bronchial asthma and chronic obstructive pulmonary disease. J Lung Pulm Respir Res. 2016;3(1):34-53. 